\title{
Mathematical Modelling of HIVIAIDS Transmission Dynamics with Drug Resistance Compartment
}

\author{
Eshetu Dadi Gurmu, Boka Kumsa Bole, Purnachandra Rao Koya \\ Department of Mathematics, Wollega University, Nekemte, Ethiopia \\ Email address: \\ eshetudadi1@gmail.com (E. D. Gurmu), abitb2012@gmail.com (B. K. Bole), drkpraophd@gmail.com (P. R. Koya)
}

\section{To cite this article:}

Eshetu Dadi Gurmu, Boka Kumsa Bole, Purnachandra Rao Koya. Mathematical Modelling of HIV/AIDS Transmission Dynamics with Drug Resistance Compartment. American Journal of Applied Mathematics. Vol. 8, No. 1, 2020, pp. 34-45. doi: 10.11648/j.ajam.20200801.16

Received: November 7, 2019; Accepted: January 2, 2020; Published: February 13, 2020

\begin{abstract}
This paper examines a mathematical modelling of HIV/AIDS transmission dynamics with drug resistance compartment. A nonlinear deterministic mathematical model for the problem is proposed using a system of ordinary differential equations. The aim of this study is to investigate the role of passive immunity and drug therapy in reducing the viral replication and transmission of the disease. The well possedness of the formulated model equations was proved and the equilibrium points of the model have been identified. In addition, the basic reproductive number that governs the disease transmission is obtained from the largest eigenvalue of the next-generation matrix. Both local and global stability of the disease free equilibrium and endemic of the model was established using basic reproduction number. The results show that the disease free equilibrium is locally asymptotically stable if the basic reproduction number is less than unity and unstable if the basic reproduction number is greater than unity. It is observed that if the basic reproduction is less than one then the solution converges to the disease free steady state i.e., disease will wipe out and thus the drug therapy is said to be successful. On the other hand, if the basic reproduction number is greater than one then the solution converges to endemic equilibrium point and thus the infectious cells continue to replicate i.e., disease will persist and thus the drug therapy is said to be unsuccessful. Sensitivity analysis of the model is performed on the key parameters to determine their relative importance and potential impact on the transmission dynamics of HIV/AIDS. Numerical results of the model show that a combination of passive immunity and drug therapy is the best strategy to reduce the disease from the community.
\end{abstract}

Keywords: HIV, Reproductive Number, Stability Analysis, Drug Therapy

\section{Introduction}

Human Immunodeficiency Virus (HIV) is the causative agent of Acquired Immunodeficiency Syndrome (AIDS). It is a disease that causes progressive failure of the immune system. HIV is an RNA retrovirus. That is, to enter a cell, HIV translates its RNA to DNA with a viral enzyme called reverse transcriptase [1]. The target cell of HIV is CD4 T cells. A healthy human body has about $1000 / \mathrm{mm}^{3}$ of CD4 T cells. When the CD4 T cells of a patient decline to $200 / \mathrm{mm}^{3}$ or below, then that person is classified as having AIDS [2]. When the CD4 $\mathrm{T}$ cells decline, they cannot mount a strong response. This results in weak responses from CTL and antibodies which cannot clear the infection [3]. HIV is transmitted primarily via unprotected sexual intercourse, contaminated blood transfusions, hypodermic needles, and from mother to child during pregnancy, delivery, or breastfeeding. There is no cure or vaccine to AIDS. However, antiretroviral (ART) treatment improves health, pro-longs life, and substantially reduces the risk of HIV transmission. More than $90 \%$ of adults in sub-Saharan Africa acquire HIV infection from unprotected sexual intercourse with infected partners [4].

Globally, new HIV infections among young women aged 15-24 years were reduced by $25 \%$ between 2010 and 2018 . This is good news, but of course it remains unacceptable that every week 6000 adolescent girls and young women become infected with HIV. The sexual and reproductive health and rights of women and young people are still too often denied. The annual number of deaths from AIDS-related illness among people living with HIV globally has fallen from a peak of 1.7 million in 2004 to 770000 in 2018. The global decline in deaths has largely been driven by progress in eastern and southern Africa, which is home to $54 \%$ of the 
world's people living with HIV. AIDS-related mortality in the region declined by $44 \%$ from 2010 to 2018 . The annual number of new infections since 2010 has declined from 2.1 million to 1.7 million in 2018 [5].

Mathematical modeling in epidemiology provides understanding of the underlying mechanisms that influence the spread of disease, and in the process, it suggests control strategies. The model formulation process clarifies assumptions, variables, and parameters. Moreover, models provide conceptual results such as thresholds, basic reproduction numbers, contact numbers, and replacement numbers. Mathematical models and computer simulations are useful experimental tools for building and testing theories, assessing quantitative conjectures, answering specific questions, determining sensitivities to changes in parameter values, and estimating the key parameters from data [6].

Many mathematical models have proved their usefulness for describing and understanding the dynamics of HIV infection. K. O. Okosun [7] presented the impact of optimal control on the treatment of HIV/AIDS and screening of unaware infectives on the transmission dynamics of the disease in a homogeneous population with constant immigration of susceptibles incorporating use of condom, screening of unaware infectives and treatment of the infected. Karrakchou et al. [8] investigated the fundamental role of chemotherapy treatment in controlling the virus reproduction in an HIV patient, while Adams et al. [9] derived HIV therapeutic strategies by formulating and analyzing an optimal control problem using two types of dynamic treatments. The author [10] develop a mathematical model for HIV/AIDS transmission has been proposed, along with a control problem in which the objective was to determine the pre-exposure prophylaxis (PrEP) strategy that minimizes the number of individuals with pre-AIDS HIV infection, balanced against the costs associated with PrEP. The paper by Mukandavire et al. [11] compares the impact of increasing condom use or HIV PrEP use among sex workers. The authors found that condom promotion interventions should remain the mainstay HIV prevention strategy for female sex workers (FSWs), with PrEP only being implemented once condom interventions have been maximized or to fill prevention gaps where condoms cannot be used. The author [12] develop a model of HIV risk and compare HIV-risk estimates before and after the introduction of PrEP to determine the maximum tolerated reductions in condom use with regular partners and clients for HIV risk not to change. With a case study of FSWs in South Africa, it is found that PrEP is likely to be of benefit in reducing HIV risk, even if reductions in condom use do occur.

So far, few mathematical studies have been undertaken to model Human Immunodeficiency Virus mathematically, but they did not considered drug resistance in their studies.

\section{Model Description and Formulation}

The model for virus dynamics consists of a system of six ordinary differential equations, including variables
$M, S, I, A, R_{S}$ and $R$ represents the Passive immunity class, susceptible class, infected class, AIDS class, drug resistance class and removed class.

i. The passive immunity individuals are produced by birth with rate $\Pi$.

ii. The susceptible individuals are increased by $\alpha$, the per unit of time rate of loss of maternal antibodies and from drug resistance compartment in which those individuals who are treated by drug therapy but did not respond to drug therapy with waning rate of $\phi$ and joined to removed class at rate $\varphi$ by losing their natural immunity.

iii. Susceptible individual may acquire HIV infection when they become into effective contact with force of infection $\lambda=\beta I / N$ where $\beta$ transmission rate constant, then the number of individuals who become infected per unit of time is equal to $\lambda=\beta I S / N$.

iv. Infected individuals move to drug resistance class at a rate $\omega$ and may progress to develop AIDS is a failure of the natural immunity at a rate $\theta$.

v. Individuals in the drug resistance class move to removed class at a rate of $\eta$ by drug therapy, with therapy efficacy of $\rho$ proportion of individuals join the removed class or join the AIDS class with $(1-\rho)$ proportion by adapting the therapy.

vi. Individuals with AIDS may die as a result of the AIDS infection at a rate $\varepsilon$.

vii. In all compartments $\mu$ is the natural mortality rate of individuals.

viii. All parameters in the model are positive.

Description of Variables and Parameters

The variables and parameters used in this model are introduced in Tables 1 and 2. Their notations and descriptions are also included.

Table 1. Description of Variables used in the model equations (1) - (6).

\begin{tabular}{ll}
\hline Variable & Description \\
\hline $\mathrm{N}(\mathrm{t})$ & The total population at time $\mathrm{t}$ \\
$\mathrm{M}(\mathrm{t})$ & Passive ImmunityCompartment \\
$\mathrm{S}(\mathrm{t})$ & Susceptible Compartment \\
$\mathrm{I}(\mathrm{t})$ & Infected Compartment \\
$\mathrm{A}(\mathrm{t})$ & AIDS Compartment \\
$\mathrm{R}_{\mathrm{s}}(\mathrm{t})$ & Drug Resistance Compartment \\
$\mathrm{R}(\mathrm{t})$ & Removed Compartment \\
\hline
\end{tabular}

Table 2. Description of parameters used in the model equations (1) - (6).

\begin{tabular}{ll}
\hline Parameter & Description \\
\hline$\Pi$ & Birth rate of passive immunity. \\
$\beta$ & Transmission rate constant. \\
$\alpha$ & Recruited rate of susceptible individuals. \\
$\omega$ & Rate of therapy. \\
$\varphi$ & Removed rate of susceptible class. \\
$\lambda$ & Force of infection. \\
$\eta$ & Removed rate of drug resistance class. \\
$\varepsilon$ & Death rate due to infection. \\
$\theta$ & Progression rate from Ito $A$. \\
$\mu$ & Natural death rate. \\
\hline
\end{tabular}

Based on the model assumptions the population flow diagram can be visualized as shown in Figure 1. 


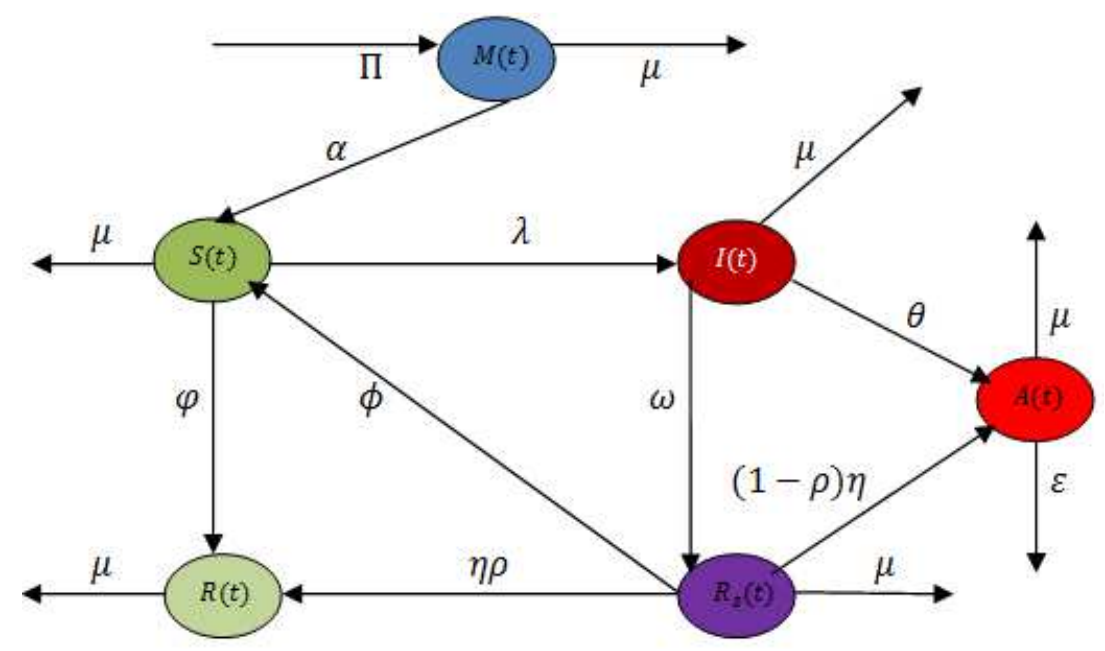

Figure 1. Schematic diagram of the model.

The population flow diagram as shown in Figure 1 can be translated into a system of six differential equations as follows:

$$
\begin{aligned}
& d M / d t=\Pi-\alpha M-\mu M \\
& d S / d t=\alpha M-\lambda+\phi R_{s}-(\varphi+\mu) S \\
& d I / d t=\lambda-(\theta+\omega+\mu) I \\
& d R_{s} / d t=\omega I-(1-\rho) \eta R_{s}-(\phi+\eta \rho+\mu) R_{S} \\
& d A / d t=(1-\rho) \eta R_{S}+\theta I-(\varepsilon+\mu) A \\
& d R / d t=\varphi S+\eta \rho R_{s}-\mu R
\end{aligned}
$$

$$
d N / d t=(d M / d t)+(d S / d t)+(d I / d t)+(d A / d t)+\left(d R_{s} / d t\right)+(d R / d t)
$$

By combining $(1-6)$ and $(7)$, we can get

$$
d N / d t=\Pi-\mu N-\varepsilon A
$$

In the absence of mortality due to AIDS (8) becomes

$$
d N / d t=\Pi-\mu N
$$

Equivalently this inequality can be expressed as a linear ordinary differential inequality as $[\mathrm{dN}(\mathrm{t}) / \mathrm{dt}]+\mu \mathrm{N}(\mathrm{t}) \leq \Pi$ giving general solution upon solving as $N(t) \leq(\Pi / \mu)+$ $c e^{-\mu t}$. But, the term $N(0)$ denotes the initial values of the respective variable i.e., $\mathrm{N}(\mathrm{t})=\mathrm{N}(0)$ at $\mathrm{t}=0$. Thus, the particular solution can be expressed as $N(t) \leq(\Pi / \mu)+$ $[\mathrm{N}(0)-(\Pi / \mu)] e^{-\mu t}$. Further, it can be observed that $N(t) \rightarrow(\Pi / \mu)$ as $t \rightarrow \infty$. That is, the total population size $N(t)$ takes off from the value $\mathrm{N}(0)$ at the initial time $\mathrm{t}=0$ and ends up with the bounded value $(\Pi / \mu)$ as the time $t$ grows to infinity. Thus, it can be concluded that $N(t)$ is bounded as $0 \leq N(t) \leq(\Pi / \mu)$. Thus, the feasible solution set of the system equation of the model enters and remains in the region:

$$
\Omega=\left\{\left(M, S, I, A, R_{S}, R\right) \in \mathfrak{R}_{+}^{6}: N \leq \Pi / \mu\right\}
$$

Therefore, the basic model is well posed epidemiologically
The non-negative initial conditions of the system of model equations (1) - (6) are denoted by $M(0)=M_{0}, S(0)=$ $S_{0}, I(0)=I_{0}, A(0)=A_{0}, R_{s}(0)=R_{s_{0}}, R(0)=R_{0}$. This system consists of six first order non-linear ordinary differential equations.

\section{Model Analysis}

\subsection{Invariant Region}

We obtained the invariant region, in which the model solution is bounded. To do this, first we considered the total human population $(N)$, Here $N=M+S+I+A+R_{S}+R$. Then, differentiating $N$ both sides with respect to $t$ leads to;

and mathematically. Hence, it is sufficient to study the dynamics of the basic model in the region $\Omega$.

\subsection{Existence of the Solution}

Lemma 1: (Existence) Solutions of the model equations (1) - (6) together with the initial conditions $M(0)>0, S(0)>$ $0, I(0)>0, A(0)>0, R_{s}(0)>0, R(0)>0$ exist in $\mathbb{R}_{+}^{6}$ i.e., the modelvariables $M(t), S(t), I(t), A(t), R_{S}(t)$ and $R(t)$ exist for all $t$ and will remain in $\mathbb{R}_{+}^{6}$.

Proof: The right hand sides of the system of equations (1) - (6) can be expressed as follows

$$
\begin{gathered}
f_{1}\left(M, \quad S, \quad I, A, \quad R_{s}, R\right)=\Pi-\alpha M-\mu M \\
f_{2}\left(M, \quad S, \quad I, A, \quad R_{s}, R\right)=\alpha M-\lambda+\phi R_{s}-(\varphi+\mu) S \\
f_{3}\left(M, \quad S, \quad I, A, \quad R_{S}, R\right)=\lambda-(\theta+\omega+\mu) I \\
\quad f_{4}\left(M, \quad S, \quad I, A, \quad R_{s}, R\right) \\
=\omega I-(1-\rho) \eta R_{s}-(\phi+\eta \rho+\mu) R_{s}
\end{gathered}
$$

$f_{5}\left(M, \quad S, \quad I, A, \quad R_{s}, R\right)=(1-\rho) \eta R_{s}+\theta I-(\varepsilon+\mu) A$

$$
f_{6}\left(M, \quad S, \quad I, A, \quad R_{s}, R\right)=\varphi S+\eta \rho R_{s}-\mu R
$$


According to Derrick and Groosman theorem, let $\Omega$ denote the region $\Omega=\left\{\left(M, S, I, A, R_{S}, R\right) \in \mathfrak{R}_{+}^{6}: N \leq \Pi / \mu\right\}$. Then equations $(1)-(6)$ have a unique solution if $\left(\partial f_{i}\right) /\left(\partial x_{j}\right), i, j=$
$1,2,3,4,5,6$ are continuous and bounded in $\Omega$. Here, $x_{1}=$ $M, x_{2}=S, x_{3}=I, x_{4}=A, x_{5}=R_{S}$ and $x_{6}=R$. The continuity and the boundedness are verified as here under:

Table 3. Continuity and boundedness of the model solution.

\begin{tabular}{ll}
\hline$\left|\left(\partial f_{1}\right) /(\partial M)\right|=|-(\alpha+\mu)|<\infty$ & $\left|\left(\partial f_{2}\right) /(\partial M)\right|=|\alpha|<\infty$ \\
$\left|\left(\partial f_{1}\right) /(\partial S)\right|=0<\infty$ & $\left|\left(\partial f_{2}\right) /(\partial S)\right|=|-(\beta I / N+\varphi+\mu)|<\infty$ \\
$\left|\left(\partial f_{1}\right) /(\partial I)\right|=0<\infty$ & $\left|\left(\partial f_{2}\right) /(\partial I)\right|=|-(\beta S / N)|<\infty$ \\
$\left|\left(\partial f_{1}\right) /(\partial A)\right|=0<\infty$ & $\left|\left(\partial f_{2}\right) /(\partial A)\right|=0<\infty$ \\
$\left|\left(\partial f_{1}\right) /\left(\partial R_{s}\right)\right|=0<\infty$ & $\left|\left(\partial f_{2}\right) /\left(\partial R_{s}\right)\right|=|\phi|<\infty$ \\
$\left|\left(\partial f_{1}\right) /(\partial R)\right|=0<\infty$. & $\left|\left(\partial f_{2}\right) /(\partial R)\right|=0<\infty$. \\
$\left|\left(\partial f_{3}\right) /(\partial M)\right|=0<\infty$ & $\left|\left(\partial f_{4}\right) /(\partial M)\right|=0<\infty$ \\
$\left|\left(\partial f_{3}\right) /(\partial S)\right|=|-(\beta I / N)|<\infty$ & $\left|\left(\partial f_{4}\right) /(\partial S)\right|=0<\infty$ \\
$\left|\left(\partial f_{3}\right) /(\partial I)\right|=|(\beta S / N)-(\theta+\omega+\mu)|<\infty$ & $\left|\left(\partial f_{4}\right) /(\partial I)\right|=\omega<\infty$ \\
$\left|\left(\partial f_{3}\right) /(\partial A)\right|=0<\infty$ & $\left|\left(\partial f_{4}\right) /(\partial A)\right|=0<\infty$ \\
$\left|\left(\partial f_{3}\right) /\left(\partial R_{s}\right)\right|=0<\infty$ & $\left|\left(\partial f_{4}\right) /\left(\partial R_{s}\right)\right|=|-[(1-\rho) \eta+(\phi+\eta \rho+\mu)]|<\infty$ \\
$\left|\left(\partial f_{3}\right) /(\partial R)\right|=0<\infty$. & $\left|\left(\partial f_{4}\right) /(\partial R)\right|=0<\infty$. \\
$\left|\left(\partial f_{5}\right) /(\partial M)\right|=0<\infty$ & $\left|\left(\partial f_{6}\right) /(\partial M)\right|=0<\infty$ \\
$\left|\left(\partial f_{5}\right) /(\partial S)\right|=0<\infty$ & $\left|\left(\partial f_{6}\right) /(\partial S)\right|=|\varphi|<\infty$ \\
$\left|\left(\partial f_{5}\right) /(\partial I)\right|=|\theta|<\infty$ & $\left|\left(\partial f_{6}\right) /(\partial I)\right|=0<\infty$ \\
$\left|\left(\partial f_{5}\right) /(\partial A)\right|=|-(\varepsilon+\mu)|<\infty$ & $\left|\left(\partial f_{6}\right) /(\partial A)\right|=0<\infty$ \\
$\left|\left(\partial f_{5}\right) /\left(\partial R_{s}\right)\right|=|(1-\rho) \eta|<\infty$ & $\left|\left(\partial f_{6}\right) /\left(\partial R_{s}\right)\right|=|\eta \rho-\mu|<\infty$ \\
$\left|\left(\partial f_{5}\right) /(\partial R)\right|=0<\infty$. & $\left|\left(\partial f_{6}\right) /(\partial R)\right|=|-\mu|<\infty$. \\
\hline
\end{tabular}

Thus, all the partial derivatives $\left(\partial f_{i}\right) /\left(\partial x_{j}\right), i, j=$ $1,2,3,4,5,6$ exist, continuous and bounded in $\Omega$. Hence, by Derrick and Groosman theorem, a solution for the model (1) -(6) exists and is unique.

\subsection{Positivity of the Solution}

We assumed that the initial condition of the model is nonnegative, and now we also will show that the solution of the model is also positive.

Theorem 1: Let $\Omega=\left\{\left(M, S, I, A, \quad R_{S}, R\right) \in \mathbb{R}_{+}^{6} ; M_{0}>\right.$ $\left.0, S_{0}>0, I_{0}>0, A_{0}>0, R_{S_{0}}>0, \mathrm{R}_{0}>0\right\} ;$ then the solutions of $\left\{M, S, I, A, R_{S}, R\right\}$ are positive for all $t \geq 0$.

Proof: Positivity is verified separately for each of the model $M(t), S(t), I(t), A(t), R_{s}(t)$ and $R(t)$.

Positivity of $M(t)$ : The model equation (1) given by $d M / d t=\Pi-\alpha M-\mu M$ can be expressed without loss of generality, after eliminating the positive terms $\Pi$ which are appearing on the right hand side, as an inequality as $d M / d t \geq-[\alpha+\mu] M$. Using variables separable method and on applying integration, the solution of the foregoing differentially inequality can be obtained as $M(t) \geq$ $M_{0}[\exp -(\alpha+\mu) t]$. Recall that an exponential function is always non-negative irrespective of the sign of the exponent, i.e., the exponential function $[\exp -(\alpha+\mu) t]$ is a nonnegative quantity. Hence, it can be concluded that $M(t) \geq 0$.

Positivity of $S(t)$ : The model equation (2) given by $d S / d t=\alpha M-\beta I S / N+\phi R_{s}-(\varphi+\mu) S$ can be expressed without loss of generality, after eliminating the positive term $\left[\alpha M+\phi R_{S}\right]$ which are appearing on the right hand side, as an inequality as $d S / d t \geq-[(\beta I / N)+(\varphi+\mu)] S$. Using variables separable method and on applying integration, the solution of the foregoing differentially inequality can be obtained as $S(t) \geq S_{0}[\exp -((\beta I / N)+(\varphi+\mu)) t]$. Recall that an exponential function is always non-negative irrespective of the sign of the exponent, i.e., the exponential function $[\exp -((\beta I / N)+(\varphi+\mu)) t]$ is a non-negative quantity. Hence, it can be concluded that $S(t) \geq 0$.

Positivity of $I(t)$ : The model equation (3) given by $d I / d t=\beta I S / N-(\theta+\omega+\mu) I$ can be expressed without loss of generality, after eliminating the positive term $[\beta I S / N]$ which are appearing on the right hand side, as an inequality as $d I / d t \geq-(\theta+\omega+\mu) I$. Using variables separable method and on applying integration, the solution of the foregoing differentially inequality can be obtained as $I(t) \geq$ $I_{0}[\exp -(\theta+\omega+\mu) t]$. Recall that an exponential function is always non-negative irrespective of the sign of the exponent i.e., the exponential function $[\exp -(\theta+\omega+\mu) t]$ is a nonnegative quantity. Hence, it can be concluded that $I(t) \geq 0$.

Positivity of $R_{s}(t)$ : The model equation (4) given by $d R_{s} / d t=\omega I-(1-\rho) \eta R_{s}-(\phi+\eta \rho+\mu) R_{s} \quad$ can be expressed without loss of generality, after eliminating the positive term $[\omega I]$ which are appearing on the right hand side, as an inequality as $d R_{s} / d t \geq-[(1-\rho) \eta+(\phi+\eta \rho+$ $\mu)] R_{s}$. Using variables separable method and on applying integration, the solution of the foregoing differentially inequality can be obtained as $R_{s}(t) \geq R_{s_{0}}[\exp -$ $((1-\rho) \eta+(\phi+\eta \rho+\mu)) t]$. Recall that an exponential function is always non-negative irrespective of the sign of the exponent, i.e., the exponential function $[\exp -$ $((1-\rho) \eta+(\phi+\eta \rho+\mu))]$ is a non-negative quantity. Hence, it can be concluded that $R_{S}(t) \geq 0$.

Positivity of $A(t)$ : The model equation (5) given by $d A / d t=(1-\rho) \eta R_{s}+\theta I-(\varepsilon+\mu) A$ can be expressed without loss of generality, after eliminating the positive term $\left[(1-\rho) \eta R_{s}+\theta I\right]$ which are appearing on the right hand side, as an inequality as $d A / d t \geq-(\varepsilon+\mu) A$. Using variables separable method and on applying integration, the solution of the foregoing differentially inequality can be obtained as $A(t) \geq$ $A_{0}[\exp -(\varepsilon+\mu) t]$. Recall that an exponential function is 
always non-negative irrespective of the sign of the exponent, i.e., the exponential function $[\exp -(\varepsilon+\mu) t]$.is a non-negative quantity. Hence, it can be concluded that $A(t) \geq 0$.

Positivity of $R(t)$ : The model equation (5) given by $d R / d t=\varphi S+\eta \rho R_{s}-\mu R$ can be expressed without loss of generality, after eliminating the positive term $[\varphi S+$ $\left.\eta \rho R_{S}\right]$ which are appearing on the right hand side, as an inequality as $d R / d t \geq-\mu R$. Using variables separable method and on applying integration, the solution of the foregoing differentially inequality can be obtained as $R(t) \geq$ $R_{0}[\exp -\mu t]$. Recall that an exponential function is always non-negative irrespective of the sign of the exponent, i.e., the exponential function $[\exp -\mu t]$. is a non-negative quantity.
Hence, it can be concluded that $R(t) \geq 0$.

Thus, the model variables $M(t), S(t), I(t), A(t), R_{S}(t)$ and $R(t)$ representing population sizes of various types of cells are positive quantities and will remain in $\mathbb{R}_{+}^{6}$ for allt.

\subsection{The Disease Free Equilibrium (DFE)}

To find the disease free equilibrium, we equated the right hand sides of model equations $(1-6)$ to zero, evaluating it at $A=I=0$ and solving for the non-infected and non-asymptomatic variables. Thus, the disease-free equilibrium point of the model equation in (1) - (6) above is given by

$$
E_{0}=\left\{M^{0}, S^{0}, I^{0}, A^{0}, R_{s}{ }^{0}, R^{0}\right\}=\{[\pi /(\alpha+\mu)],[\alpha \pi /(\alpha+\mu)(\varphi+\mu)], 0,0,0,[\varphi \alpha \pi / \mu(\alpha+\mu)(\varphi+\mu)]\}
$$

\subsection{The Basic Reproduction Number $\left(\mathfrak{R}_{0}\right)$}

In this section we obtained the threshold parameter that governs the spread of a disease which is called the basic reproduction number is obtained. To obtain the basic reproduction number, we used the next-generation matrix method so that it is the spectral radius of the next-generation matrix [13].

The model equations are rewritten starting with newly infective classes:

$$
F=\left[\begin{array}{cc}
{[\beta \alpha \mu /(\alpha+\mu)(\varphi+\mu)]} & 0 \\
0 & 0
\end{array}\right] \text { and } V=\left[\begin{array}{cc}
\theta+\omega+\mu & 0 \\
-\theta & \varepsilon+\mu
\end{array}\right]
$$

It can be verified that the matrix $V$ is non-singular as its determinant $\operatorname{det}[V]=(\theta+\omega+\mu)(\varepsilon+\mu)$ is non-zero and after some algebraic computations its inverse matrix is constructed as

$$
F V^{-1}=\left[\begin{array}{cc}
{[\beta \alpha \mu /(\alpha+\mu)(\varphi+\mu)]} & 0 \\
0 & 0
\end{array}\right]\left[\begin{array}{c}
{[1 /(\theta+\omega+\mu)]} \\
{[\theta /(\varepsilon+\mu)(\theta+\omega+\mu)]}
\end{array}\right.
$$

$$
V^{-1}=\left[\begin{array}{cc}
{[1 /(\theta+\omega+\mu)]} & 0 \\
{[\theta /(\varepsilon+\mu)(\theta+\omega+\mu)]} & {[1 /(\varepsilon+\mu)]}
\end{array}\right]
$$

The product of the matrices $F$ and $V^{-1}$ can be computed as:

$$
\left[\begin{array}{c}
0 \\
{[1 /(\varepsilon+\mu)]}
\end{array}\right]=\left[\begin{array}{cc}
{[\beta \alpha \mu /(\alpha+\mu)(\varphi+\mu)(\varphi+\mu)]} & 0 \\
0 & 0
\end{array}\right]
$$

the threshold value or the basic reproductive number. Thus, it can be concluded that the reproduction number of the model is $\mathfrak{R}_{0}=[\beta \alpha \mu /(\alpha+\mu)(\varphi+\mu)(\varphi+\mu)]$. the basic reproduction number $\mathfrak{R}_{0}$ by taking the spectral radius of the matrix $F V^{-1}$. Thus, the eigenvalues are computed by evaluating $\operatorname{det}\left[F V^{-1}-\psi I\right]=0$ or equivalently solving

$$
\left|\begin{array}{cc}
{[\beta \alpha \mu /(\alpha+\mu)(\varphi+\mu)(\varphi+\mu)]-\psi} & 0 \\
0 & -\psi
\end{array}\right|=0
$$

It reduces to the quadratic equation for $\psi$ as $-\psi[[\beta \alpha \mu /(\alpha+\mu)(\varphi+\mu)(\varphi+\mu)]-\psi]=0$ giving the two eigenvalues as $\psi_{1}=[\beta \alpha \mu /(\alpha+\mu)(\varphi+\mu)(\varphi+$ $\mu)], \psi_{2}=0$.

However, the dominant eigenvalue here is $\psi_{1}=$ $[\beta \alpha \mu /(\alpha+\mu)(\varphi+\mu)(\varphi+\mu)]$ and is the spectral radius as

\subsection{Local Stability of Disease Free Equilibrium}

Theorem 2: The disease free equilibrium point $E_{0}$ of the system (1) - (6) is locally asymptotically stable if $\Re_{0}<1$ and unstable if $\Re_{0}>1$.

Proof: To proof this theorem first we obtain the Jacobian matrix of system (10) at the disease free equilibrium $E_{0}$ as follows:

$$
J\left(E_{0}\right)=\left[\begin{array}{cccccc}
-a & 0 & 0 & 0 & 0 & 0 \\
\alpha & -(\varphi+\mu) & -\Re_{0} & 0 & \phi & 0 \\
0 & 0 & \mathfrak{R}_{0}-b & 0 & 0 & 0 \\
0 & 0 & \theta & -(\varepsilon+\mu) & (1-\rho) \eta & 0 \\
0 & 0 & \omega & 0 & -[(1-\rho) \eta+c] & 0 \\
0 & \varphi & 0 & 0 & \eta \rho & -\mu
\end{array}\right]
$$


Now, the eigenvalues of $J\left(E_{0}\right)$ are required to be found. The characteristic equation $\operatorname{det}\left[J\left(E_{0}\right)-\psi I\right]=0$ is expanded and simplified as follows:

$$
\begin{gathered}
\left|\begin{array}{cccccc}
-a-\psi & 0 & 0 & 0 & 0 & 0 \\
\alpha & -(\varphi+\mu)-\psi & -\Re_{0} & 0 & \phi & 0 \\
0 & 0 & \mathfrak{R}_{0}-b-\psi & 0 & 0 & 0 \\
0 & 0 & \theta & -(\varepsilon+\mu)-\psi & (1-\rho) \eta & 0 \\
0 & 0 & \omega & 0 & -[(1-\rho) \eta+c]-\psi & 0 \\
0 & \varphi & 0 & 0 & \eta \rho & -\mu-\psi
\end{array}\right|=0 \\
{[-a-\psi][-(\varphi+\mu)-\psi]\left[\Re_{0}-b-\psi\right][-(\varepsilon+\mu)-\psi][-[(1-\rho) \eta+c]-\psi][-\mu-\psi]=0}
\end{gathered}
$$

Thus, the five eigenvalues of the matrix are determined as

$$
\begin{gathered}
\psi_{1}=-\psi \\
\psi_{2}=-(\varphi+\mu) \\
\psi_{3}=-\left(\Re_{0}-b\right) \\
\psi_{4}=-(\varepsilon+\mu) \\
\psi_{5}=-[(1-\rho) \eta+c] \\
\psi_{6}=-\mu
\end{gathered}
$$

Where

$$
a=(\alpha+\mu), b=(\theta+\omega+\mu), c=(\phi+\eta \rho+\mu)
$$

It can be observed that all the eigenvalues $\psi_{1}, \psi_{2}, \psi_{3}, \psi_{4}, \psi_{5}$ and $\psi_{6}$ are absolutely negative quantities. Therefore, it is concluded that the DFE $E_{0}$ of the system of differential equations (1) - (6) is locally asymptotically stable if $\Re_{0}<1$ and unstable if $\Re_{0}>1$.

\subsection{Global Stability of the DFE $E_{0}$}

Theorem 3: The disease free equilibrium point $E_{0}$ of the model is globally asymptotically stable if $\Re_{0}<1$ and unstable if $\Re_{0}>1$.

Proof: To prove the global asymptotic stability of the DFE we use the method of Lyapunov function. Systematically, we define a Lyapunov function $L$ such that:

$$
L=[1 /(\theta+\omega+\mu)] I
$$

Then

$$
\begin{gathered}
A^{*}=[1 /(\varepsilon+\mu)]\left[\left((1-\rho) \eta \omega \lambda^{*}\right) / b\right. \\
R_{S}^{*}=\left[\omega \lambda^{*}\right] /[b(1-\rho) \eta+b c] \\
R^{*}=\left[\varphi S^{*}+\left[\eta \rho \omega \lambda^{*}\right] /[b(1-\rho) \eta+b c]\right][1 / \mu]
\end{gathered}
$$

Here

$$
a=(\alpha+\mu), b=(\theta+\omega+\mu), c=(\phi+\eta \rho+\mu) .
$$

On substituting the expression for $I^{*}$ into the force of infection, that is, $\lambda^{*}=\left[\beta I^{*}\right] /[N]$, characteristic polynomial of force of infection is obtained as

$$
p\left(\lambda^{*}\right)=D_{1} \lambda^{*}
$$

$$
\begin{gathered}
d L / d t=[1 /(\theta+\omega+\mu)] d I / d t \\
=[1 /(\theta+\omega+\mu)][(\beta S I / N)-(\theta+\omega+\mu) I] \\
=[(\beta \alpha \mu) /(\theta+\omega+\mu)(\alpha+\mu)(\varphi+\mu)-1] I \\
=\left[\Re_{0}-1\right] I
\end{gathered}
$$

So $d L / d t \leq 0$, if $\Re_{0}<1$. Furthermore, $d L / d t=0$ if $I=0$ or $\Re_{0}=1$. From this we see that, $\left\{M^{0}, S^{0}, 0,0, R_{S}^{0}, R^{0}\right\}$ is the only singleton in $\left\{\left(M^{0}, S^{0}, I^{0}, A^{0}, R_{s}^{0}, R^{0}\right) \in\right.$ $\Omega: d L / d t=0\}$. Therefore by the principle of (LaSalle, 1976), DFE is globally asymptotically stable if $\Re_{0} \leq 1$.

\subsection{The Endemic Equilibrium}

Endemic equilibrium point $E_{1}$ is a steady state solution where the disease persists in the community. For the existence and uniqueness of endemic equilibrium $E_{1}=$ $\left\{M^{*}, S^{*}, I^{*}, A^{*}, R_{S}^{*}, R^{*}\right\}$ its coordinates should satisfy the conditions $E_{1}=\left\{M^{*}, S^{*}, I^{*}, A^{*}, R_{S}^{*}, R^{*}\right\} \neq 0$, where $\mathrm{M}_{0}>$ $0, \mathrm{~S}_{0}>0, \mathrm{I}_{0}>0, \mathrm{~A}_{0}>0, \mathrm{R}_{\mathrm{S}_{0}}>0$, and $\mathrm{R}_{0}>0$. The endemic equilibrium point is obtained by setting left hand sides of equations of the system (1) - (6) to zero. We then solved for state variables in terms of the force of infection, $\lambda^{*}$ and obtain the following;

$$
\begin{gathered}
M^{*}=\Pi / a \\
S^{*}=\left[[\alpha \Pi] /[a]+\left[\phi \omega \lambda^{*}\right] /[b(1-\rho) \eta+b c]\right. \\
\left.-\lambda^{*}\right][1 /(\varphi+\mu)] \\
I^{*}=\lambda^{*} / b
\end{gathered}
$$


endemic equilibrium we use the method of Lyapunov functions. Define

$$
\begin{gathered}
L\left(M^{*}, S^{*}, I^{*}, A^{*}, R_{S}^{*}, R^{*}\right)=\left[M-M^{*}-M^{*} \ln \left(M^{*} / M\right)\right]+\left[S-S^{*}-S^{*} \ln \left(S^{*} / S\right)\right]+ \\
{\left[I-I^{*}-I^{*} \ln \left(I^{*} / I\right)\right]+\left[A-A^{*}-A^{*} \ln \left(A^{*} / A\right)\right]+\left[R_{S}-R_{S}^{*}-R_{S}^{*} \ln \left(R_{S}^{*} / R_{S}\right)\right]+\left[R-R^{*}-R^{*} \ln (R)\right]}
\end{gathered}
$$

By direct calculating the derivative of $L$ along the solution $(1-6)$ we have

$$
\begin{aligned}
& d L / d t=\left[\left(M-M^{*}\right) / M\right] d M / d t+\left[\left(S-S^{*}\right) / S\right] d S / d t+\left[\left(I-I^{*}\right) / I\right] d I / d t+ \\
& {\left[\left(A-A^{*}\right) / A\right] d A / d t+\left[\left(R_{S}-R_{S}^{*}\right) / R_{S}\right] d R_{S} / d t+\left[\left(R-R^{*}\right) / R\right] d R / d t,} \\
& =\left[\left(M-M^{*}\right) / M\right][\Pi-\alpha M-\mu M]+\left[\left(S-S^{*}\right) / S\right]\left[\alpha M-\lambda+\phi R_{S}-(\varphi+\mu) S\right]+\left[\left(I-I^{*}\right) / I\right][\lambda-(\theta+\omega+\mu) I] \\
& +\left[\left(A-A^{*}\right) / A\right]\left[(1-\rho) \eta R_{S}+\theta I-(\varepsilon+\mu) A\right]+\left[\left(R_{S}-R_{S}^{*}\right) / R_{S}\right]\left[\omega I-(1-\rho) \eta R_{S}-(\phi+\eta \rho+\mu) R_{S}\right] \\
& +\left[\left(R-R^{*}\right) / R\right]\left[\varphi S+\eta \rho R_{s}-\mu R\right] \\
& =\left[1-M^{*} / M\right][\Pi-\alpha M-\mu M]+\left[1-S^{*} / S\right]\left[\alpha M-\lambda+\phi R_{S}-(\varphi+\mu) S\right]+\left[1-I^{*} / I\right][\lambda-(\theta+\omega+\mu) I] \\
& +\left[1-A^{*} / A\right]\left[(1-\rho) \eta R_{S}+\theta I-(\varepsilon+\mu) A\right]+\left[1-R_{S}^{*} / R_{S}\right]\left[\omega I-(1-\rho) \eta R_{s}-(\phi+\eta \rho+\mu) R_{s}\right] \\
& +\left[1-R^{*} / R\right]\left[\varphi S+\eta \rho R_{S}-\mu R\right] \\
& d L / d t=\Pi+(1-\rho) \eta R_{s}^{*}+\alpha M^{*}+\varphi S^{*}+(\theta+\omega) I^{*}+\varepsilon A^{*}+(\phi+\eta \rho) R_{S}^{*}+\left[M^{*}+S^{*}+I^{*}+R_{S}^{*}+R^{*}\right] \mu \\
& +\left(\lambda+\phi R_{s}\right)\left[S^{*} / S\right] \\
& -\left[\left(\Pi M^{*} / M\right)+\left(\alpha \mathrm{M} S^{*} / S\right)+\left(\lambda I^{*} / I\right)+\left((1-\rho) \eta \mathrm{R}_{\mathrm{s}} A^{*} / A\right)+\left(\theta \mathrm{I} A^{*} / A\right)+\left(\omega \mathrm{I} R_{S}^{*} / R_{S}\right)\right. \\
& \left.+\left(\left(\varphi \mathrm{S}+\eta \rho \mathrm{R}_{\mathrm{s}}\right) R^{*} / R\right)+\varepsilon A+\left(M+S+I+A+R_{S}+R\right) \mu\right]
\end{aligned}
$$

Thus collecting positive and negative terms together we obtain

$$
\begin{gathered}
d L / d t=\Pi+(1-\rho) \eta R_{s}^{*}+\alpha M^{*}+\varphi S^{*}+(\theta+\omega) I^{*}+\varepsilon A^{*}+(\phi+\eta \rho) R_{s}^{*}+N^{*} \mu+ \\
\left(\lambda+\phi R_{s}\right)\left[S^{*} / S\right]-\left[\left(\Pi M^{*} / M\right)+\left(\alpha \mathrm{M} S^{*} / S\right)+\left(\lambda I^{*} / I\right)+\left((1-\rho) \eta \mathrm{R}_{\mathrm{s}} A^{*} / A\right)+\left(\theta \mathrm{I} A^{*} / A\right)+\left(\omega \mathrm{I} R_{s}^{*} / R_{s}\right)\right. \\
\left.+\left(\left(\varphi S+\eta \rho \mathrm{R}_{\mathrm{s}}\right) R^{*} / R\right)+\varepsilon A+N \mu\right]
\end{gathered}
$$

$$
d L / d t=Q-K
$$

Here, $Q=\Pi+(1-\rho) \eta R_{s}^{*}+\alpha M^{*}+\varphi S^{*}+(\theta+\omega) I^{*}+\varepsilon A^{*}+(\phi+\eta \rho) R_{s}^{*}+N^{*} \mu+\left(\lambda+\phi R_{s}\right)\left[S^{*} / S\right]$

$$
\begin{gathered}
K=\left[\left(\Pi M^{*} / M\right)+\left(\alpha \mathrm{M} S^{*} / S\right)+\left(\lambda I^{*} / I\right)+\left((1-\rho) \eta \mathrm{R}_{\mathrm{s}} A^{*} / A\right)+\left(\theta \mathrm{I} A^{*} / A\right)+\left(\omega \mathrm{I} R_{S}^{*} / R_{S}\right)+\left(\left(\varphi \mathrm{S}+\eta \rho \mathrm{R}_{\mathrm{s}}\right) R^{*} / R\right)+\varepsilon A+N \mu\right] \\
N=M+S+I+A+R_{S}+R \text { and } N^{*}=M^{*}+S^{*}+I^{*}+R_{S}^{*}+R^{*}
\end{gathered}
$$

Thus if $Q<K$, then $d L / d t \leq 0$. Noting that $d L / d t=0$ if

\begin{tabular}{|c|c|c|}
\hline Parameter & Value & Reference \\
\hline$\Pi$ & 15 & assumed \\
\hline$\mu$ & 0.02 & assumed \\
\hline$\beta$ & 4 & assumed \\
\hline$\varphi$ & 0.03 & assumed \\
\hline$\alpha$ & 0.8 & assumed \\
\hline$\omega$ & 0.8 & assumed \\
\hline$\varepsilon$ & 0.01 & assumed \\
\hline$\eta$ & 0.05 & assumed \\
\hline$\phi$ & 0.04 & assumed \\
\hline$\theta$ & 0.5 & assumed \\
\hline$\rho$ & 0.48 & assumed \\
\hline
\end{tabular}
and only if $M=M^{*}, S=S^{*}, I=I^{*}, A=A^{*}, R_{s}=R_{s}^{*}, R=$ $R^{*}$. Therefore, the largest compact invariant set in $\left\{\left(M^{*}, S^{*}, I^{*}, A^{*}, R_{S}^{*}, R^{*}\right) \in \Omega: d L / d t=0\right\}$ is the singleton $E_{1}$ is the endemic equilibrium of the system $(1-6)$. By LaSalle's invariant principle (LaSalle's, 1976), it implies that $E_{1}$ is globally asymptotically stable in $\Omega$ if $Q<K$.

\section{Numerical Simulation}

In this section, the numerical simulation study of model equations (1) - (6) is carried out using the software DE Discover 2.6.4. To conduct the study, a set of meaningful values are assigned to the model parameters. These values are either taken from literature or assumed. Using the parameter values given in Table 3 and the initial conditions

$$
\begin{gathered}
M(0)=1000, S(0)=800, I(0)=600 \\
, R_{S}(0)=500, A(0)=100
\end{gathered}
$$

and $R(0)=400$ in the model equations (1) - (6) a simulation study is conducted and the results are given in the following Figures.

Table 4. Parameter values used in Numerical Simulations.

Figure 2 shows that the basic reproduction number with respect to drug therapy. It is evident that drug therapy played a major role in reducing viral replication and stability to be achieved at $\mathfrak{R}_{0}<1$.

Figure 3 shows that the basic reproduction number with passive immunity. It is evident that passive immunity plays a role in reducing viral replication. Clearly, for $\Re_{0}<1$, the stability of disease free equilibrium is achievable in the presence of passive immunity. 


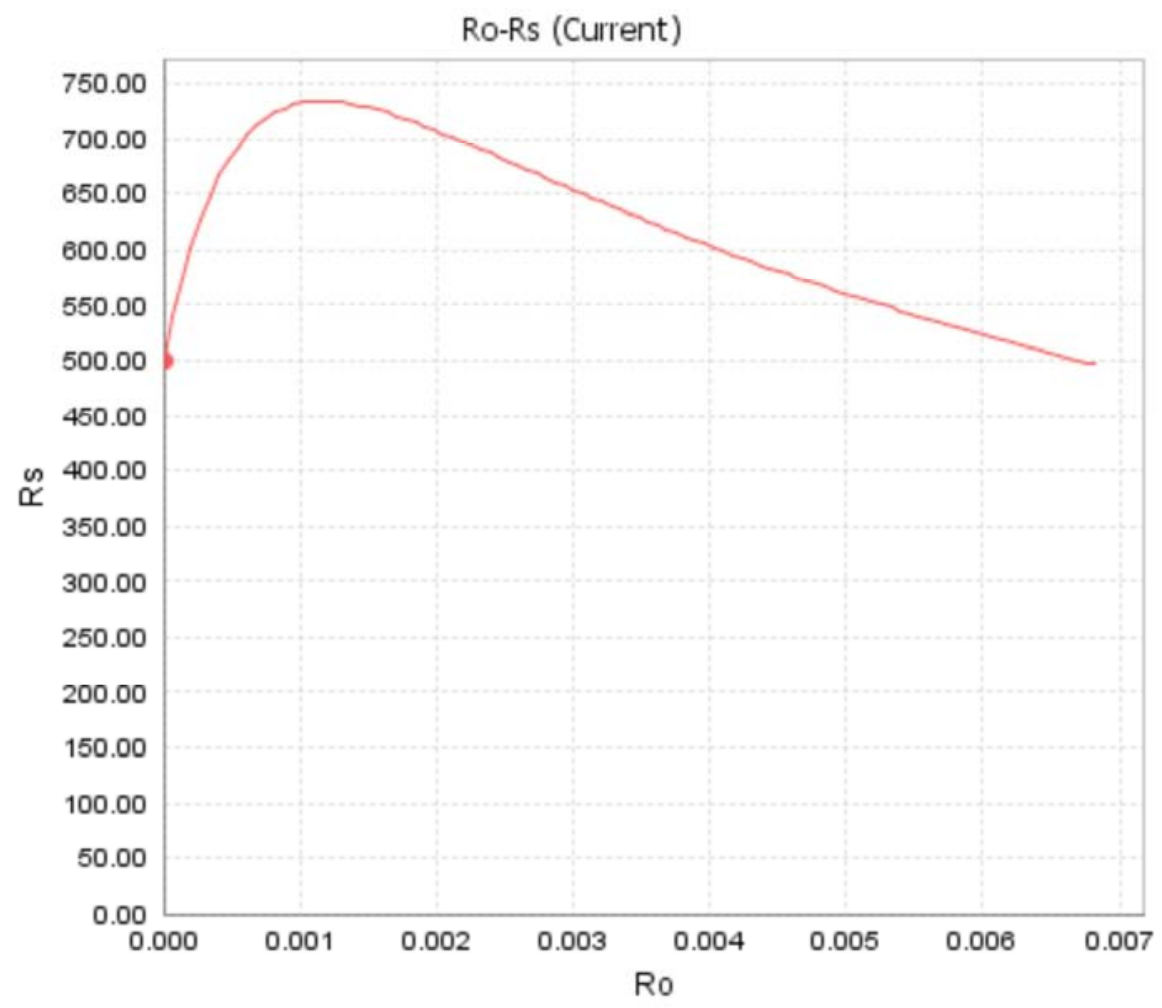

- Ro-Rs

Figure 2. Figure showing $\mathfrak{R}_{0}$ with respect to Drug Resistance.

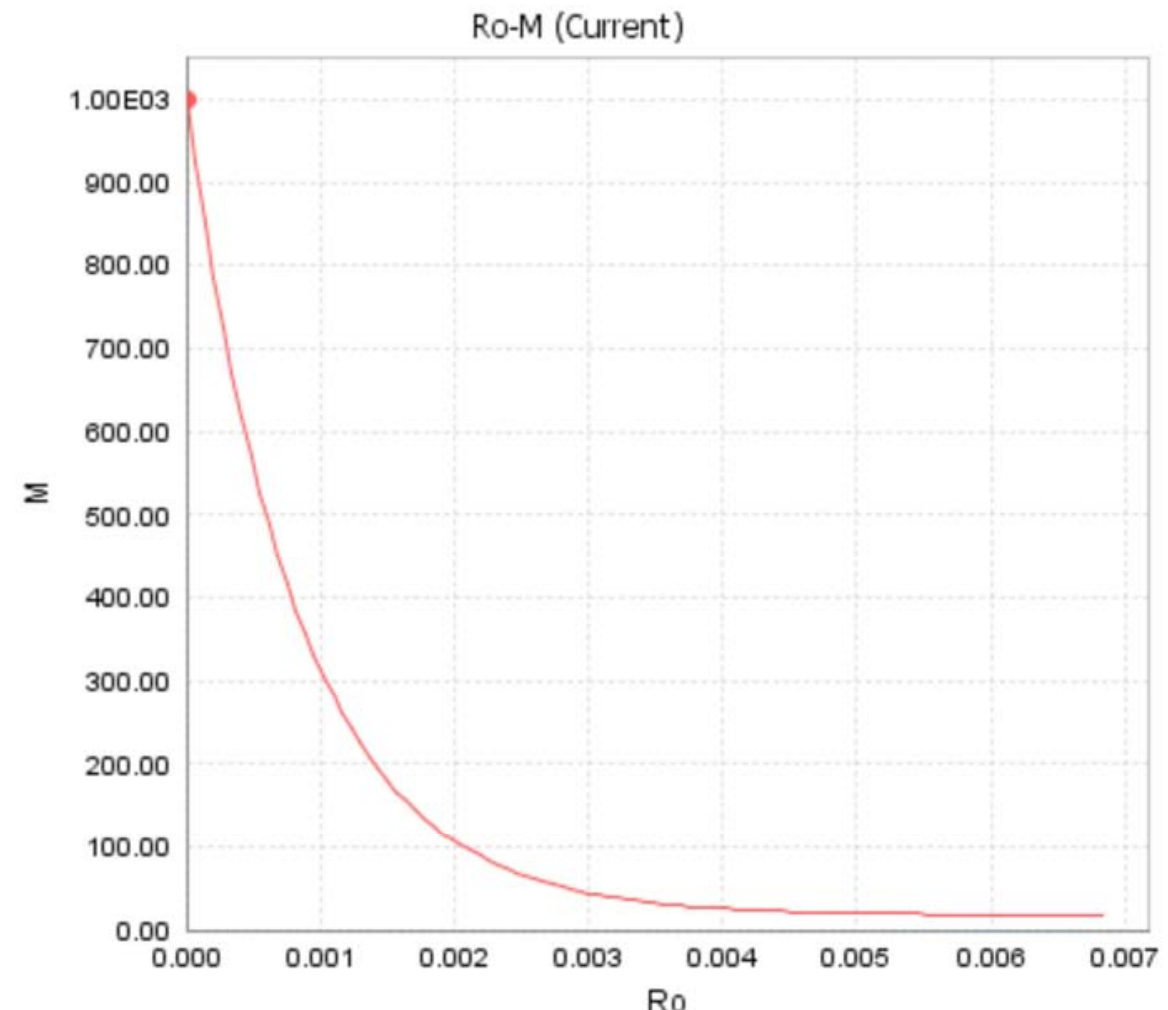

Ro

- Ro-M

Figure 3. Figure showing $\Re_{0}$ with respect to Passive Immunity. 
Figure 4 shows that the number of susceptible individuals, infected individuals and AIDS individuals are decreases slowly due to the presence of passive immunity and drug therapy. With $\Re_{0}=0.156$ and the transmission $\beta=0.53$, implies that passive immunity and drug therapy can reduce the viral replication and transmission of the disease in the population when $\mathfrak{R}_{0}<1$.

Figure 5 shows that the number of infected individuals and
AIDS individuals rises slowly to a value slightly above $200 / \mathrm{mm}^{3}$. This implies that passive immunity and drug therapy played a major role in reducing the viral replication and transmission.

Figure 6 shows that the number of are increases when there were no passive immunity and drug therapy. But the number of susceptible individuals decreases, implying that most of susceptible individuals are infected and removed.

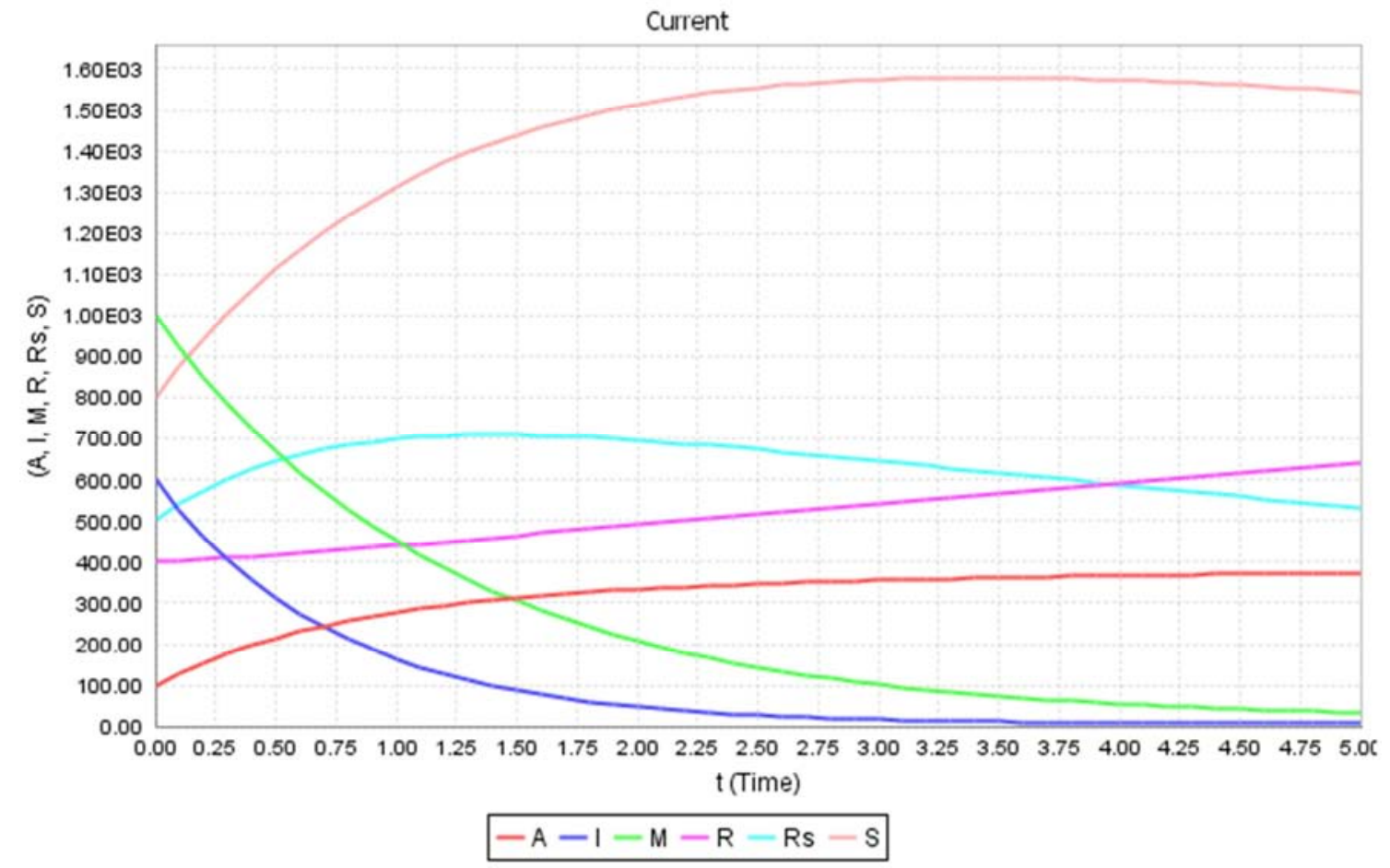

Figure 4. Simulation results when $\Re_{0}<1$.

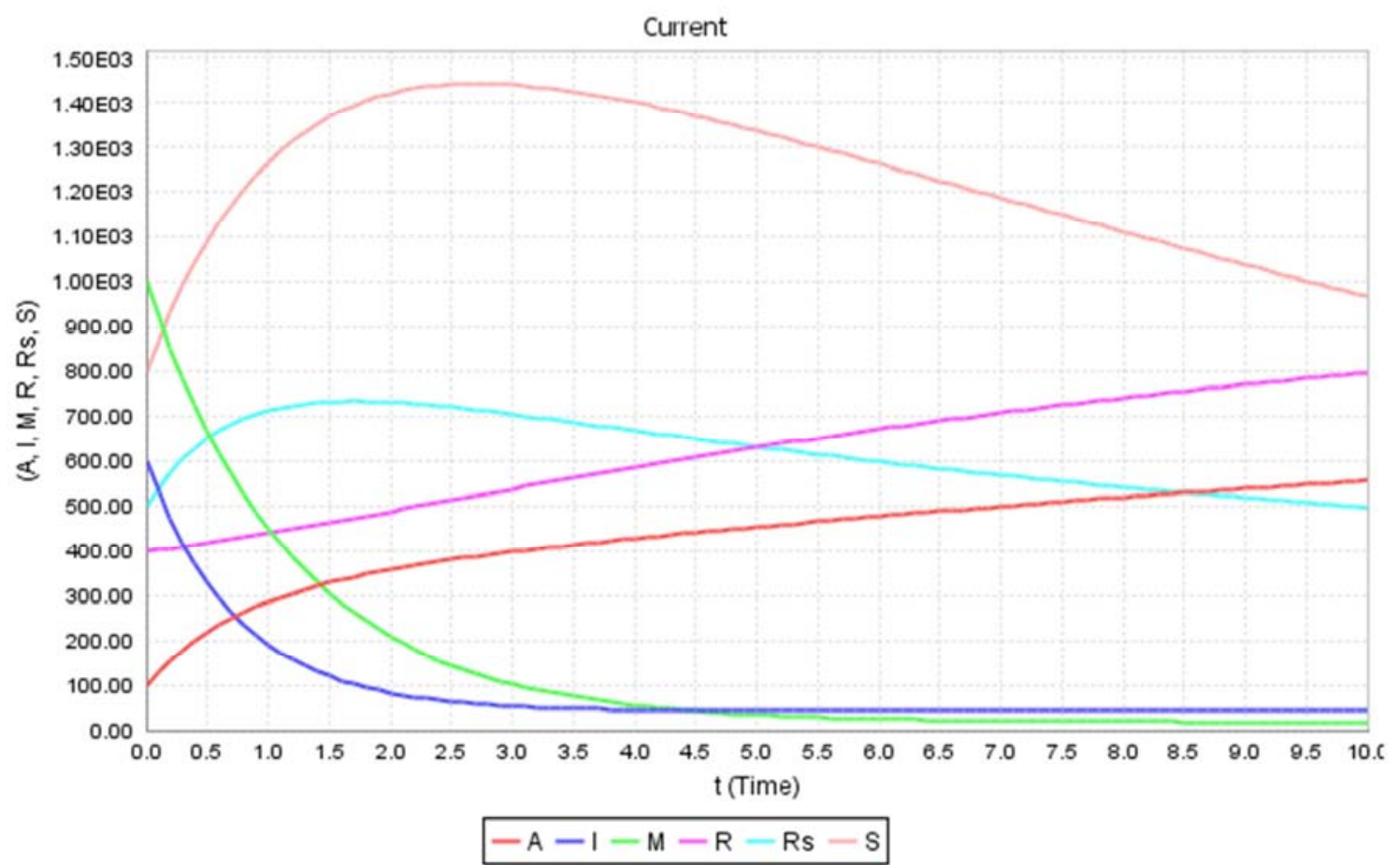

Figure 5. Simulation results when $\Re_{0}>1$. 


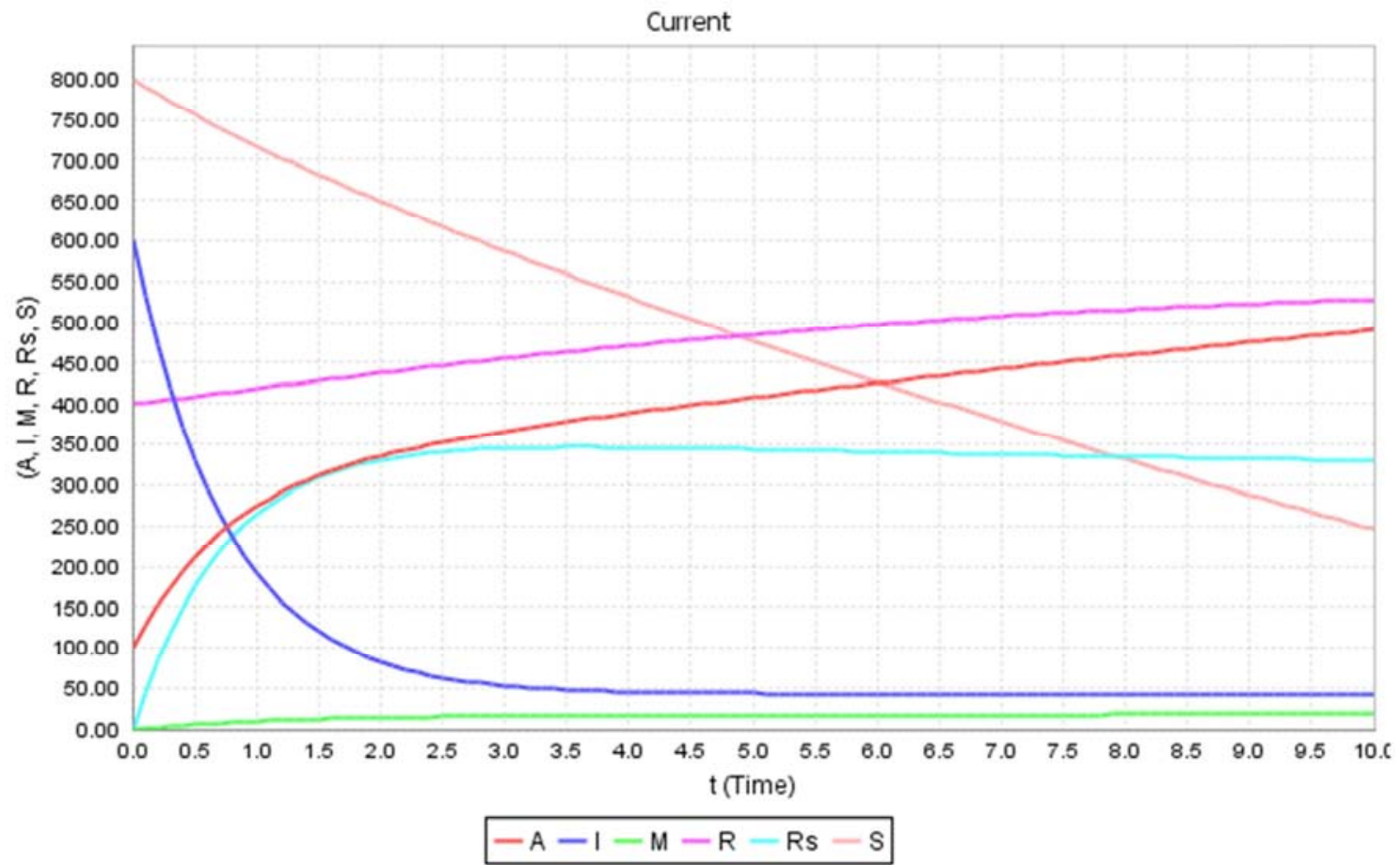

Figure 6. Simulation results without passive immunity and drug therapy.

\section{Sensitivity Analysis}

We carried out sensitivity analysis in order to determine the relative significance of model parameters on disease transmission. The analysis will enable us to find out parameters that have high impact on the basic reproduction number and which should be targeted by intervention strategies. We perform sensitivity analysis by calculating the sensitivity indices of the basic reproduction number in order to determine whether HIV can be spread in the population or not. These indices tell us how crucial each parameter is on

$$
\begin{aligned}
& \Upsilon_{\beta}^{R_{0}}=\left[\partial R_{0} / \beta\right] \times\left[\beta / R_{0}\right]=1 \\
& \Upsilon_{\alpha}^{R_{0}}=\left[\partial R_{0} / \alpha\right] \times\left[\alpha / R_{0}\right]=\mu /(\alpha+\mu) \\
& \Upsilon_{\mu}^{R_{0}}=\left[\partial R_{0} / \mu\right] \times\left[\mu / R_{0}\right] \\
& =\left[\begin{array}{c}
(\alpha+\mu)(\varphi+\mu)(\theta+\omega+\mu)- \\
\mu[(\varphi+\mu)(\theta+\omega+\mu)+(\alpha+\mu)(\theta+\omega+\mu)+(\alpha+\mu)(\varphi+\mu)]
\end{array}\right] /[(\alpha+\mu)(\varphi+\mu)(\theta+\omega+\mu)] \\
& \Upsilon_{\varphi}^{R_{0}}=\left[\partial R_{0} / \varphi\right] \times\left[\varphi / R_{0}\right]=-\varphi /(\varphi+\mu) \\
& \Upsilon_{\theta}^{R_{0}}=\left[\partial R_{0} / \theta\right] \times\left[\theta / R_{0}\right]=-\theta /(\theta+\omega+\mu) \\
& \Upsilon_{\omega}^{R_{0}}=\left[\partial R_{0} / \omega\right] \times\left[\omega / R_{0}\right]=-\omega /(\theta+\omega+\mu)
\end{aligned}
$$

the transmission of the HIV. To investigate which parameters in the model system (1) - (6) have high impact on the $\mathfrak{R}_{0}$, we ply the approach presented by [14].

$$
\mathfrak{R}_{0}=[\beta \alpha \mu /(\alpha+\mu)(\varphi+\mu)(\theta+\omega+\mu)] .
$$

Since $\mathfrak{R}_{0}$ depends only on six parameters, we derive an atical expression for its sensitivity to each parameter using the normalized forward sensitivity index as by Chitnis [15] as follows:
Table 5. Sensitivity indices Table.

\begin{tabular}{ll}
\hline Parameter Symbol & Sensitivity indices \\
\hline$\beta$ & +1 \\
$\mu$ & 0.5608 \\
$\alpha$ & 0.02439 \\
$\theta$ & -0.37878 \\
$\varphi$ & -0.6 \\
$\omega$ & -0.6060 \\
\hline
\end{tabular}

The sensitivity indices of the basic reproductive number with respect to main parameters are arranged orderly in Table 5. Those parameters that have positive indices i.e. $\beta, \mu$ and $\alpha$ show that they have great impact on expanding the disease in the community if their values are increasing. Due to the reason that the basic reproduction number increases as their values increase, it means that the average number of secondary cases of infection increases in the community. 
Furthermore, those parameters in which their sensitivity indices are negative i.e. $\theta, \varphi$ and $\omega$ have an influence of minimizing the burden of the disease in the community as their values increase while the others are left constant. And also as their values increase, the basic reproduction number decreases, which leads to minimizing then endemicity of the disease in the community.

Furthermore, sensitivity testing of $M, S, I, R S, R$ shows the
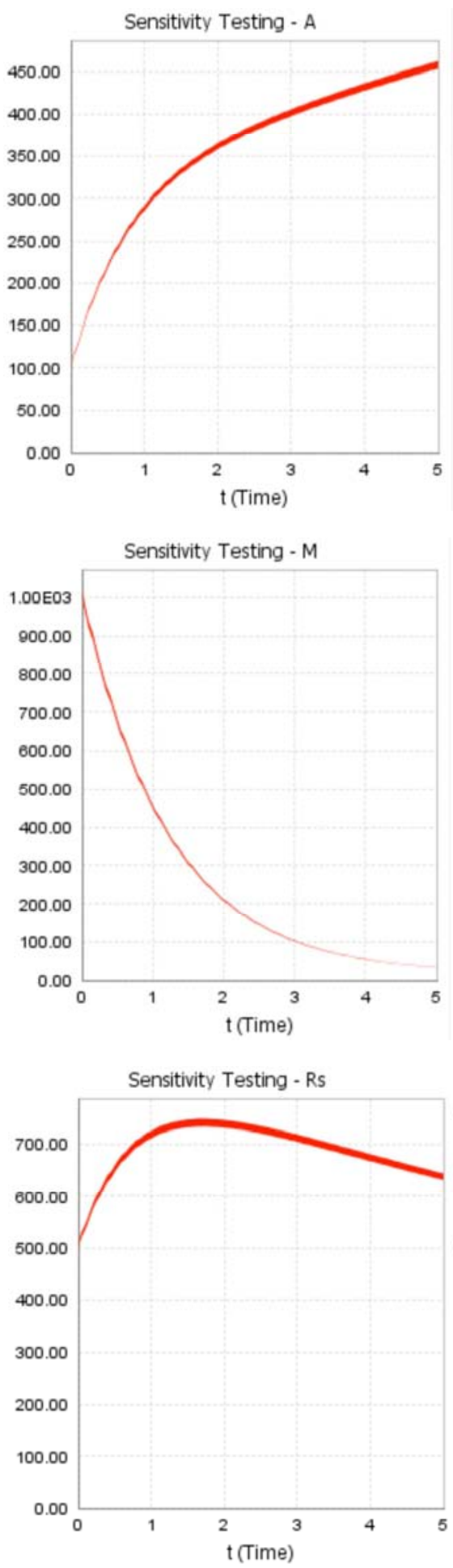

sensitivity of each compartment. This explain that susceptible individuals, drug resistance individuals and removed compartment are medium, i.e. which leads to minimizing the endemicity of the disease in the community. Furthermore, those variables $I$ and $A$ (in some interval) in which their status are maximum, i.e. which have an influence of minimizing the burden of the disease in the community, but passive immunity has minimum impact.
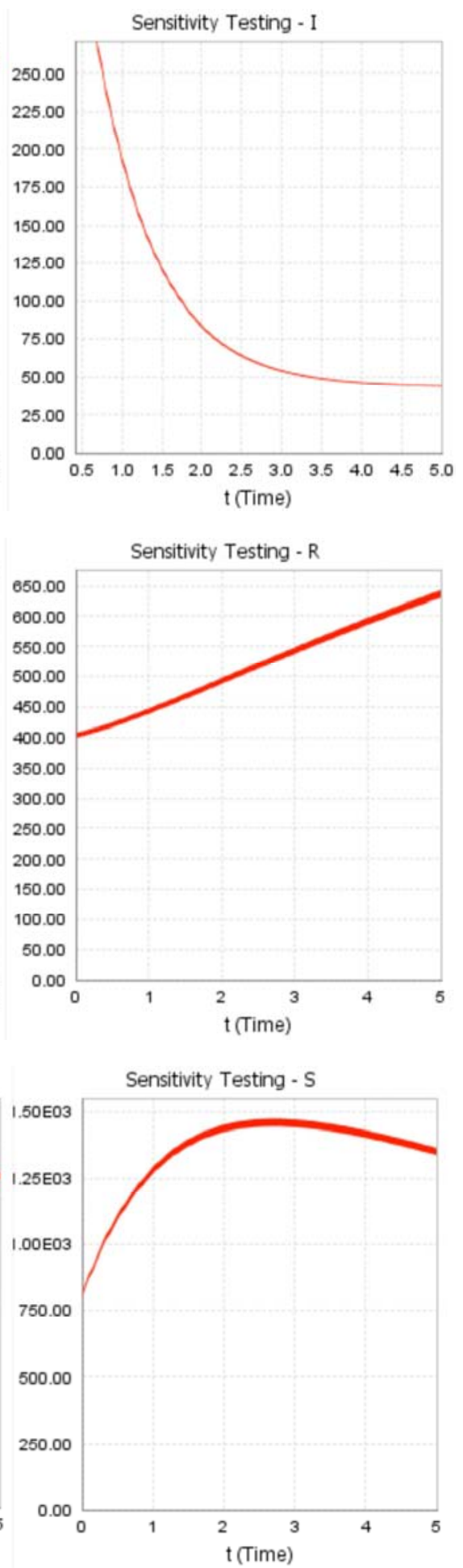

Minimum — Maximum — Median — 10th Quantile — 90th Quantile 


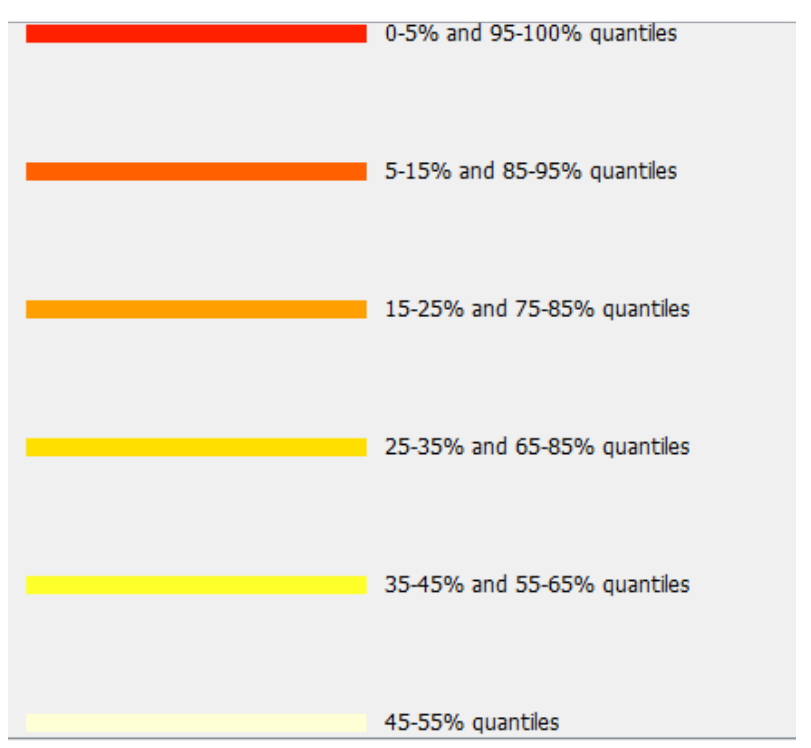

Figure 7. Sensitivity testing of $M, S, I, A, R s, R$.

\section{Discussions and Conclusions}

In this study, we proposed a mathematical modelling of HIV/AIDS transmission dynamics with drug resistance compartment. The qualitative analysis of the model shows that there exists a domain where the model is epidemiologically and mathematically well-posed. The stability analysis of the model was investigated using the threshold parameter that governs the disease transmission. The disease free and endemic equilibrium points was obtained and their stabilities are investigated. It was established that the disease free equilibrium is locally stable if the basic reproduction number $\mathfrak{R}_{0}<1$ and unstable if the basic reproduction number $\Re_{0}>1$. The endemic equilibrium, which exist only when $\Re_{0}>1$, is globally asymptotically stable. The solution of the model equation is numerically supplemented and the sensitivity analysis of the model equation is analyzed to determine which parameter has high impact on the transmission of the diseases.

\section{References}

[1] Wodarz, D. (2007). Killer Cell Dynamics, Mathematical and Computational Approaches to Immunology. Springer Verlag, New York.

[2] Nowak, M. A., and May, R. M. (2000). Virus Dynamics: Mathematical Principles of Immunology and Virology. Oxford University Press, Oxford, U. K.

[3] Nowak, M. A., and Bangham, C. R. M. (1996). Population dynamics of immune responses to persistent viruses. Science $272,5258,74$.

[4] N. Rom, S. B. Markowitz (2007). Environmental and Occupational Medicine, Lippincott Williams \& Wilkins.

[5] UNAIDSDATA (2019).

[6] Robert J. Smith, Jing Li, Jun Mao and Beni Sahai (2013).
Using within-host Mathematical Modelling to predict the long-term outcome of Human Papillomavirus Vaccines. volume 38 Canadian Applied Mathematics Quarterly, 21 (2).

[7] K. O. Okosun, O. D. Makinde, I. Takaidza (2013). Impact of optimal control on the treatment of HIV/AIDS and screening of unaware infectives. Applied Mathematical Modelling 37, 3802-3820.

[8] Karrakchou, M. Rachik, S. Gourari (2006). Optimal control and infectiology: application to an HIV/ AIDS model, Appl. Math. Comput. 177, 807-818.

[9] B. M. Adams, H. T. Banks, Kwon Hee-Dae, T. T. Hien T (2004). Dynamic multidrug therapies for HIV: Optimal and STI control approaches. Mathematical Biosciences and Engineering. 1 and 2, 223-241.

[10] Silva, CJ, Torres, DF (2017). Modeling and optimal control of HIV/AIDS prevention through PrEP. arXiv: 1703. 06446.

[11] Mukandavire, Z, Mitchell, KM (2016). Comparing the impact of increasing condom use or HIV pre-exposure prophylaxis (PrEP) use among female sex workers. Epidemics 14, 62-70.

[12] Grant, H, Mukandavire, Z, Eakle, R, Prudden, H, Gomez, GB, Rees, H, Watts, C (2017). When declines in condom use while using are PrEP a concern Modelling insights from a Hillbrow. South Africa case study. J. Int. AIDS Soc. 20 (1), 21744.

[13] Eshetu Dadi Gurmu, Purnachandra Rao Koya (2019). Sensitivity Analysis and Modeling the Impact of Screening on the Transmission Dynamics of Human Papilloma Virus (HPV). American Journal of Applied Mathematics. 7 (3), pp. 70-79.

[14] Eshetu Dadi Gurmu and Purnachandra Rao Koya (2019). Impact of Chemotherapy treatment of SITR Compartmentalization and Modeling of Human Papilloma Virus (HPV). IOSR Journal of Mathematics (IOSR-JM). 15 (3), Pp. 17-29.

[15] Chitnis, N., Hyman, J. M., and Cusching, J. M. (2008). Determining important Parameters in the spread of malaria through the sensitivity analysis of a mathematical Model. Bulletin of Mathematical Biology. 70 (5), 1272-12. 